\title{
La Variable Personalidad en el Estudio de la Psicoterapia: ¿Medida de Resultado o Proceso? ${ }^{1}$
}

\author{
Personality as a Variable in Psychotherapy Research: \\ Outcome or Process Measurement?
}

\author{
Alemka Tomicic Suñer \\ Pontificia Universidad Católica de Chile \\ Claudio Martínez Guzmán \\ Pontificia Universidad Católica de Chile
}

(Rec: 07 agosto 2008 Acep: 26 abril 2009)

\begin{abstract}
Resumen
La investigación sobre efectividad psicoterapéutica y el estudio de la personalidad han ocurrido de manera más o menos paralela. Actualmente es posible plantear al menos dos puntos de convergencia: el estudio del efecto de la psicoterapia sobre el cambio de la personalidad y la investigación de los efectos de las variables de personalidad de los pacientes sobre el proceso psicoterapéutico. En el presente artículo se exponen algunos avances de la investigación de proceso-resultado en psicoterapia que incluyen la personalidad como variable. En particular, se presenta el problema del cambio de la personalidad y se revisan algunas investigaciones en psicoterapia que la han incorporado como una medida de resultado o de proceso. Se concluye y discute el valor de la noción de personalidad en la investigación de la psicoterapia, integrando asuntos teóricos, prácticos y metodológicos.
\end{abstract}

Palabras Claves: Investigación en psicoterapia, personalidad, cambio.

\begin{abstract}
Psychotherapeutic effectivity research and personality research have developed in parallel ways. Nowadays, it is possible to identify two convergent perspectives: the study of the effect of the psychotherapy over personality change and the study of the effects of patients' personality variables over the psychotherapeutic process. In this paper some advances of process-outcome psychotherapy research, which includes personality as a variable, are shown. Specifically, the issue of personality change is presented and empirical studies, which include personality as an outcome or process measurement, are reviewed. The value of the concept of personality for psychotherapy research is discussed integrating theoretical, practical and methodological topics.
\end{abstract}

Key words: Psychotherapy research, personality, change.

1 Correspondencia a e-mail: atomicic@uc.cl; comartin@uc.cl 
Las primeras aproximaciones a la investigación sobre los efectos de la psicoterapia se focalizaron en comprender la manera como ésta permitía transformar las estructuras de personalidad así como los síntomas de los pacientes. Así, no era extraño encontrar dentro de los criterios para definir una terapia como exitosa la observación de "un cambio de la personalidad analíticamente aceptable" junto a una mejoría de la sintomatología (Bergin \& Lambert, 1978). Asimismo, las reflexiones sobre las condiciones necesarias para la efectividad del proceso terapéutico, consideraban el cambio como una transformación de la personalidad (e.g. en el año 1957, Carl Roger publica un artículo en el Journal of Consulting Psychology, titulado "The necesssary and sufficient conditions of therapeutic personality change", en el cual plantea que la relación terapéutica constituye la principal condición para el cambio constructivo de la personalidad).

De acuerdo con Lambert y Supplee (1997), la creciente popularidad del conductismo en las décadas de los 60 y 70 , implicó un drástico cambio en los métodos empleados para evaluar los resultados de la psicoterapia, a la vez que conllevó a una transformación del estatus y la importancia de los tratamientos basados y orientados a la modificación de la personalidad. De esta forma, cobró mayor relevancia la posibilidad de dar cuenta de cambios observables de manera directa, con lo cual el principal criterio para definir una terapia como exitosa fue el de la disminución de la sintomatología. De aquí en adelante, los desarrollos de la investigación de la efectividad en psicoterapia y la medición de la personalidad han ocurrido en vías más o menos paralelas. Sin embargo, actualmente es posible dar cuenta de dos puntos de encuentro entre ambas líneas de indagación: por un lado, si nos preguntamos sobre el valor del constructo de personalidad como medida de resultado de la psicoterapia, es decir, sobre el efecto de la psicoterapia en términos de transformación de la personalidad y, por el otro lado, si nos preguntamos sobre el valor del constructo de personalidad como medida de proceso de la psicoterapia, vale decir, sobre los efectos de las variables de personalidad de los pacientes sobre el proceso y los resultados psicoterapéuticos.

En lo que sigue, se exponen brevemente los avances actuales de la investigación del cambio en psicoterapia discutiendo el lugar que ha tenido el constructo de la personalidad en dichos avances. Posteriormente, se discute la concepción de cambio según diferentes modelos de personalidad actualmente en uso, para luego presentar una revisión de algunas investigaciones que han establecido la relación entre psicoterapia y personalidad como resultado y como proceso. Finalmente, se concluye con una discusión sobre el valor y el sentido de la noción de personalidad en la investigación de la psicoterapia, incluyendo asuntos teóricos, prácticos y metodológicos.

\section{Investigación del cambio en psicoterapia y personalidad.}

Los esfuerzos más importantes de la investigación en psicoterapia han sido los destinados a precisar la relación entre psicoterapia y resultado (outcome) (Lambert \& Barley, 2001).

La investigación sobre los resultados de la psicoterapia ha demostrado insistentemente que ésta es efectiva en la reducción de los síntomas o en la resolución de los problemas específicos que presentan los pacientes. Sin embargo, las variables de la personalidad no han sido usadas con el mismo éxito para dar cuenta de su efectividad. Lambert y Supplee (1997) han afirmado que la investigación de resultado en psicoterapia y la investigación en personalidad han continuado desarrollos en esferas completamente separadas.

El cambio de la personalidad como resultado de la psicoterapia ha sido estudiado escasamente e incluso ha sido considerado irrelevante para los propósitos o metas de muchos estudios. Algunos factores que han contribuido a esta situación son: a) la popularidad de los enfoques conductuales y de la estandarización de la medición de la personalidad; b) la creencia derivada de perspectivas humanistas que considera al diagnóstico y la evaluación como prácticas que no aportan a la relación de ayuda con las personas que buscan la psicoterapia; c) la creciente popularidad de los enfoques cognitivos con un énfasis en constructos acotados; d) la creencia respecto que los test de personalidad no operan bien y cuentan con coeficientes de validez imprecisos debido a que centran su medición en los rasgos de personalidad, excluyendo las variables situacionales; e) el exceso de tiempo requerido para la medición y d) el énfasis actual en la realización de tratamientos breves, orientados a las crisis y cuyo objetivo es la restauración de los niveles anteriores de funcionamiento (Lambert \& Supplee, 1997).

Por otro lado, se cuenta con suficiente evidencia que demuestra la efectividad de la psicoterapia-como función de cambio y/o reducción de síntomas- (Asay \& Lambert, 1999; Lambert \& Barley, 2001; Wampold, Ahn, Coleman, 2001; Messer \& Wampold, 2002; Wampold, 2005). Sin embargo, permanecen interrogantes respecto a los aspectos que contribuyen a dicha efectividad (Roth \& Fonagy, 2006). En este sentido, la pregunta por estos aspectos ha movilizado hasta la actualidad innumerables estudios sobre el proceso terapéutico y su relación con los resultados (Krause, 2005), estudios en los cuales las variables de personalidad de los pacientes han tenido mayor protagonismo - en tanto factores de cambio- que en los estudios de resultado.

Como ya es ampliamente sabido, los resultados de las investigaciones orientadas al proceso no han demostrado aportes diferenciales o específicos de los diferentes enfoques terapéuticos y de las técnicas que de ellos se derivan. 
En el meta-análisis de los resultados de un importante conjunto de investigaciones en el área realizado por Lambert y Barley (2001), los autores concluyeron que las mejorías del paciente eran explicadas sólo en un $15 \%$ por los factores "técnicos", siendo el $85 \%$ restante explicado por el cambio extraterapéutico (40\%), factores comunes (30\%) y las expectativas del paciente o efecto placebo (15\%).

Si bien no se dispone de evidencia lo suficientemente robusta como para afirmar que los factores específicos no son los responsables de los resultados de la psicoterapia, no es menos cierto que la evidencia disponible es, en su mayoría, consistente con los modelos que sugieren la potencia de los factores inespecíficos. De esta manera, se ha planteado el problema de asumir la importancia de factores específicos ignorando las restantes fuentes de variabilidad que afectan los resultados en la psicoterapia. Por ejemplo, el contexto, que incluye las características del paciente (Wampold, 2005).

Específicamente, se ha encontrado que el cambio extraterapéutico, vale decir, la remisión espontánea -que explicaría un porcentaje importante de los resultados de la psicoterapia- depende de la severidad y complejidad de la condición, su cronicidad, la presencia de un trastorno de personalidad subyacente y la naturaleza, fortaleza y calidad del soporte social (Lambert \& Barley, 2001). En este mismo sentido, Sprenkle y Blow (2004) reportan que los factores del paciente corresponden a su personalidad y que los elementos extraterapéuticos refieren a los componentes de la vida y el entorno del mismo que tienen un impacto en la ocurrencia del cambio. Esto en conjunto con el porcentaje de varianza explicada por las expectativas del paciente, presta apoyo a la conclusión que los resultados de la psicoterapia se encuentran fuertemente impactados por las características individuales de los pacientes y las circunstancias fuera del contexto terapéutico (e.g. Tallman \& Bohart, 1999).

\section{Personalidad y cambio}

La manera como definimos los aspectos esenciales de la personalidad tiene tremendas implicancias para considerar las posibilidades de cambio o estabilidad de la misma (Tickle, Heatherton, Wittenberg, 2001). Asimismo, las posibilidades de cambio o estabilidad de la personalidad podrían tener implicancias respecto de las maneras de estudiarla y analizarla en psicoterapia: como un aspecto o variable de resultado o como un aspecto o variable del proceso.

Tickle et al. (2001), describen tres formas de definición de la personalidad y sus implicancias para la consideración del cambio en la personalidad.

En la perspectiva de los rasgos básicos, la personalidad es conceptualizada como un pequeño conjunto de rasgos básicos y latentes, alrededor de los cuales las dimensiones de la personalidad pueden ser categorizadas (Tickle et al., 2001). De acuerdo con Millon, Meagher y Grossman (2001), esta manera de concebir la personalidad responde a una aproximación inductiva-dimensional en la cual no se asumen compromisos teóricos previos y por lo tanto el modelo de la personalidad que se construye depende de los distintos dominios de la personalidad en los cuales se encuentren datos disponibles. Relacionado con esta aproximación empiricista y debido a la existencia de múltiple evidencia a favor de la estabilidad a lo largo del tiempo de los rasgos básicos de personalidad, este tipo de conceptualización prácticamente no considera la posibilidad de que ésta pueda ser modificada (Tickle et al., 2001).

Por su parte, desde un enfoque en el cual se concibe a la persona como totalidad, la personalidad es definida en términos más amplios que los rasgos básicos, incluyendo por ejemplo, motivaciones, estilos personales, roles, esquemas, actitudes, autoconcepto, etc. Empleando esta definición de personalidad, se obtiene mayor evidencia a favor del cambio en ésta, sobre todo cuando se la concibe de manera jerárquica (Meyer, 2004; Tickle et al., 2001). Además, estos modelos de personalidad se caracterizan por ser desarrollados desde un modelo teórico que determina ciertos principios subyacentes que organizan los contenidos de todos los dominios de la personalidad y que permiten prefigurar o hipotetizar mecanismos de cambio (Millon et al., 2001).

Finalmente, las perspectivas situadas o situacionales, plantean que la personalidad no es estable y que su consistencia en el tiempo es más bien una ilusión. Esta se debería principalmente al efecto de la consistencia en el entorno. Experimentos de elicitación de comportamientos y la evidencia de la vida cotidiana (e.g. las interacciones diarias) son las principales fuentes empíricas que avalan estas perspectivas (Tickle et al., 2001). En este caso, la posibilidad de cambio de la personalidad será en distintos grados función de las alternativas de modificación del entorno.

Estas maneras de definir la personalidad y las implicancias para las posibilidades de cambio o estabilidad de la misma, aportan ciertas dimensiones de análisis para examinar el papel de la personalidad en la investigación en psicoterapia: como una variable de resultado o como una variable del proceso.

Con respecto a la personalidad como una medida de resultado, es importante destacar que el estudio de su estabilidad o cambio depende de algunos aspectos metodológicos. En primer lugar, con qué frecuencia es medida o evaluada es un factor a considerar toda vez que la estabilidad de la personalidad disminuye cuando los intervalos de medición aumentan. En segundo lugar, la manera cómo es definida y medida la personalidad. Y finalmente, aún cuando los promedios grupales sugieran estabilidad en la personalidad, es posible que una minoría logre cambios. En este sentido, es importante la consideración de aproximaciones ideográficas 
además de las nomotéticas para el estudio del cambio en la personalidad (Tickle et al., 2001).

Ogles, Lambert, Weight y Payne (1990) analizaron la práctica de evaluación empleada en investigaciones de resultado de la psicoterapia para la agarofobia entre 1966 y 1988. Uno de los resultados interesantes fue el grado en el cual diferentes mediciones y diferentes tipos de medición reflejaron el tamaño de los cambios ocurridos como resultado de la psicoterapia. Los investigadores estimaron el tamaño del efecto de varias mediciones y de varias clases de mediciones. La comparación reflejó la diferencia entre mediciones, más que las diferencias entre distintos tipos de tratamientos, poblaciones de pacientes y diferencias procedurales. Como resultado, las mediciones que indicaban cambios en síntomas mostraban un mayor tamaño de efecto que aquellas que mostraban cambios en la personalidad.

Por un lado, resultados como estos refuerzan el valor de emplear mediciones específicas de síntomas por sobre los inventarios de personalidad para demostrar la potencia de los tratamientos y, por el otro, sugieren que las medidas de personalidad aplicadas al estudio de la psicoterapia, suelen dar cuenta de los aspectos durables que hacen de la personalidad un fenómeno estable y que la medición tradicional de la personalidad puede no ser sensible a los atributos "estables" de la personalidad que pudiesen ser modificados por la psicoterapia (Lambert \& Supplee, 1997).

Con respecto a la relación entre personalidad y psicoterapia como una medida del proceso, se han realizado un número considerable de estudios que relacionan personalidad con los resultados de la terapia. Algunas de las variables de personalidad incluidas en estos estudios han sido: nivel o grado de perturbación, situación vital, sistemas de apoyo, expectativas de mejorías, motivación al tratamiento, fortaleza del ego, habilidad para la relación terapéutica, estructuras cognitivas, neuroticismo, entre otras (Garfield, 1994). Como es posible apreciar, algunas de estas variables se corresponden con conceptualizaciones más amplias de la personalidad que la de los rasgos básicos, incluyendo dimensiones intrapsíquicas de mayor complejidad e integración y aspectos situacionales que determinarían ciertas características o expresiones de la personalidad.

Una de las variables del paciente más frecuentemente evaluada en relación con los resultados de la psicoterapia ha sido el grado de trastorno, integración o patología en la personalidad. Otra variable de personalidad que ha sido mencionada frecuentemente por terapeutas psicoanalíticos como predictora del resultado de la terapia ha sido la "fortaleza del ego". Por su parte, la inteligencia es otra variable que ha recibido atención por parte de algunos investigadores, sosteniendo que si la psicoterapia es un proceso de aprendizaje o involucra algún aprendizaje, algún mínimo de inteligencia podría ser requerido (Garfield, 1994).

Ahora bien, la posibilidad de establecer relaciones entre las variables de personalidad de los pacientes y los resultados de la psicoterapia, también está sujeta a algunas consideraciones metodológicas. Por ejemplo, en el estudio de la relación entre el nivel de trastorno de personalidad del paciente y el resultado debido a la terapia, es posible enumerar una serie de problemas en las mediciones: la confiabilidad de los métodos de evaluación de la personalidad, la variedad de criterios para establecer los tipos de resultados (p.e. jueces expertos versus autorreporte) y los tipos de muestras (homogeneidad versus heterogeneidad de diagnósticos) (Garfield, 1994).

\section{Estudios de resultados: el efecto de la psicoterapia en la personalidad}

En relación con la estabilidad de la personalidad, dos estudios prospectivos sobre los resultados (outcome) de trastornos de personalidad -el Collaborative Longitudinal Personality Study (CLPS) y el McLean Study of Adult Development (MSAD) - han mostrado la remisión a lo largo del tiempo de pacientes que cumplían con los criterios para cuatro tipos de trastornos de personalidad (Skodol, et. al., 2005; Zanarini, et. al., 2005).

Paris (2005) señala que estos resultados, al mostrar que muchas patologías de la personalidad declinan a lo largo del tiempo, cuestionan la validez del Eje II como sistema clasificatorio de los trastornos de personalidad puesto que asume la cronicidad de los mismos. Además, es importante considerar que los pacientes que participan de estos tipos de estudios pueden ser más condescendientes (característica que puede contribuir a la mejoría a lo largo del tiempo), a diferencia de las poblaciones clínicas que en general son más difíciles y menos complacientes. A pesar de lo anterior, la conclusión respecto que la patología tiende a declinar en el tiempo es insoslayable.

Ahora bien, en relación con la manera de concebir la personalidad, ambos estudios cuestionan una noción unidimensional de la misma. En este sentido, Skodol et. al. (2005, CLP), reconoce que no es posible concluir a partir de su estudio que los trastornos de personalidad muestran "remisiones", puesto que, si bien los pacientes no cumplen con los criterios diagnósticos, continúan presentando bajos niveles de funcionamiento. Es decir, presentan menos síntomas, pero mantienen rasgos desadaptativos que los afectan en sus relaciones y actividades laborales. Zanarini et al. (2005, MSAD) también señalan que los pacientes de su muestra no alcanzan una recuperación completa.

Así, considerando los problemas de funcionamiento que los pacientes mantienen luego de la remisión del desorden de personalidad, ambos autores sugieren que las categorías actuales para clasificar los trastornos de personalidad son híbridos de rasgos (que muestran consistencia a lo largo del tiempo) y síntomas (que tienden a remitir en el tiempo) (Paris, 2005). 
Por su parte, el estudio de Lingiardi, Shedler y Gazzilio (2005) ilustra una aproximación ideográfica para la investigación de la relación entre psicoterapia y cambio de la personalidad. Los autores investigaron el cambio de la personalidad en una paciente con diagnóstico de trastorno de personalidad en tratamiento psicoterapéutico. Con el fin de superar la escisión entre las aproximaciones clínicas y empíricas de evaluación de la personalidad, los autores emplearon el SWAP-200 (Shedler-Westwern Assessment Procedure-200, un set de 200 sentencias descriptivas de la personalidad) que, a juicio de los mismos, permitía conciliar la perspectiva nomotética centrada en los signos y síntomas de las escalas estructuradas de diagnóstico y la ideográfica centrada en los procesos mentales más propia de la indagación clínica. Respecto de los resultados, este estudio mostró que, después de dos años de psicoterapia, con sesiones tres veces a la semana, la paciente no cumplía con los criterios para ser diagnosticada con un trastorno de personalidad. Los cambios más importantes en la paciente fueron una disminución en los puntajes para los rasgos limítrofes, histriónicos y antisociales alcanzando el nivel de la población normal, una disminución significativa en el uso de drogas, el mantenimiento de una relación de pareja estable, un trabajo por más de un año y el establecimiento de relaciones más balanceadas con los miembros de su familia. No obstante estos promisorios resultados, los autores no pudieron concluir respecto de la eficacia del tratamiento en general o de una intervención en particular, pues se trató de un caso único.

La investigación de la efectividad de la psicoterapia ha mostrado que los síntomas, comportamientos y la personalidad cambian de manera diferente, como una función del tiempo y el curso de la terapia. De esta manera, las diferentes aproximaciones metodológicas para la investigación de los resultados de la psicoterapia nos hacen esperar distintos niveles o grados de cambio dependiendo de las áreas de funcionamiento que son evaluadas (Fowler, et. al., 2004).

Al respecto, Howard et al. (1996) mostraron la relación entre la cantidad de tratamiento y la mejora sintomática por medio del modelo dosis-efecto. Los autores plantearon una relación lineal entre el número de sesiones y el tipo de mejoría de los pacientes siguiendo un modelo de tres fases secuenciales que implican distintos niveles y grados de cambio: remoralización, remediación y rehabilitación. La primera se lograría en las sesiones iniciales y tendría relación con la capacidad de los pacientes de movilizarse y activar sus recursos de enfrentamiento. La segunda requeriría de unas 16 sesiones y se focalizaría en el alivio sintomático. Finalmente, la fase de rehabilitación se focalizaría en el cambio de los comportamientos problemáticos y desadaptativos para el establecimiento de nuevas formas de tratar con variados aspectos de la vida. Los autores sostienen que esta última fase muestra cierta gradualidad en el cambio y que su duración depende de la severidad de dichos problemas o estilos desadaptativos de funcionamiento.

Perry, Banon y Ianni (1999) estimaron que los pacientes con trastornos de personalidad requerían al menos 2,2 años de tratamiento para obtener un $75 \%$ de recuperación, definida ésta como no cumplir con los criterios del DSM-IV para un trastorno de personalidad.

Más recientemente, Fowler et al. (2004), informaron mejorías en el funcionamiento de los pacientes que estudiaron. Estas mejorías fueron consistentes con el modelo de fases de cambio de la psicoterapia: a los 16 meses de tratamiento las mayores mejorías fueron observadas a nivel de la sintomatología, el funcionamiento en las relaciones interpersonales y el funcionamiento en el trabajo y los estudios. Estos cambios fueron seguidos por mejorías relativas en funciones más estables de la personalidad tales como la cualidad de las representaciones objetales, la comprensión social, nivel de desorden de pensamiento y procesos de agresiones primarias.

Estos estudios permiten ilustrar el estado de la investigación del cambio de la personalidad como efecto de la psicoterapia, señalándonos alcances y limitaciones de la misma. El primer alcance es que han mostrado que la personalidad puede cambiar en el tiempo y a lo largo del curso de la psicoterapia, y que la posibilidad de dar cuenta de esos cambio puede encontrar limitaciones si se opta por estudiar psicoterapias de corta duración y si los instrumentos de medición no consideran a la personalidad como un constructo multidimensional. Una segunda consideración es que, debido a la falta de investigaciones que incorporen grupos control, aún es difícil sostener con certeza que este cambio pueda explicarse de manera significativa por la psicoterapia y aún menos precisar técnicas específicas que operen como mecanismos de dichos cambios.

Al respecto, en un estudio en el cual fueron evaluados los cambios en la personalidad de pacientes tratados por trastorno depresivo mayor, los investigadores examinaron 599 pacientes (empleando el Modelo de Cinco Factores) que fueron asignados a distintas formas de tratamiento de un programa de seis meses de duración que combinaba farmacoterapia con psicoterapia. Los investigadores encontraron que los cambios en los rasgos de personalidad de los pacientes fueron explicados en un pequeño porcentaje por los cambios en la severidad del cuadro depresivo, y que en general, los rasgos permanecieron relativamente estables, a excepción del rasgo de estabilidad emocional, independiente de la severidad del estado depresivo y del tipo de intervención psicofarmacológica (De Fruyt, Van Leeuwen, Bagby, Rolland \& Rouillon, 2006).

Otro avance en el sentido de precisar técnicas específicas que operen como mecanismos de cambio, es un estudio realizado por Mayer (2004) quien, empleando un listado de 52 técnicas que teóricamente posibilitaban cambios en diferentes áreas de la personalidad, consultó a jueces expertos 
(terapeutas) de manera de establecer la confiabilidad de dicha clasificación. De esta manera evaluó la existencia de un acuerdo confiable entre los jueces respecto de la influencia teórica de dichas técnicas de cambio sobre áreas específicas de la personalidad (Mayer, 2004).

El interés especial en el estudio del trastorno de personalidad limítrofe en comparación con otros trastornos de personalidad representa otro aporte para el establecimiento de técnicas terapéuticas para propiciar el cambio de los trastornos de personalidad (Day \& Bryan, 2007; Fonagy, Roth \& Higgits, 2005). La creciente evidencia de resultados positivos en el tratamiento de pacientes con personalidad limítrofe muestra la viabilidad de programas de investigación de resultados en psicoterapia para los trastornos de personalidad, sobre todo cuando se ha enfatizado que estos grupos de pacientes suelen presentar problemas de vinculación y compromiso con el tratamiento. Al respecto, Roth \& Fonagy (2006) señalan que no se deben subestimar los problemas prácticos asociados a la implementación de estos estudios en particular, pero a la vez parece razonable continuar desarrollando estas líneas de investigación considerando el impacto social que suelen conllevar estos trastornos.

\section{Estudios de proceso: el efecto de la personalidad en la psicoterapia}

Se han realizado numerosas investigaciones buscando relacionar las variables de personalidad de los pacientes con las características del proceso terapéutico y los resultados del mismo (Garfield, 1994; Clarkin \& Levi, 2004). Algunas de las variables de personalidad estudiadas han incluido la presencia de trastornos de personalidad (considerado como un rasgo extremo dentro del continuo de la normalidad y el trastorno), las expectativas del paciente, la fuerza del ego y la capacidad de mentalización (Bateman \& Fonagy, 2008; Clarkin \& Levi; 2004; Garfield, 1994).

Una de las variables de personalidad más estudiadas en relación con su influencia en los resultados de la psicoterapia ha sido la presencia de algún trastorno de personalidad. Al respecto, se ha planteado que los individuos con trastornos de personalidad severos obtienen menos beneficios de las terapias, vale decir, muestran resultados más pobres una vez finalizado dicho tratamiento (Garfield, 1994). En este sentido, se ha estudiado el trastorno de personalidad como una variable moderadora de los resultados de la psicoterapia (Clarkin \& Levi, 2004; Hoglend et. al., 2008).

Van Beek y Verheul (2008) realizaron una investigación con el objetivo de examinar la asociación entre trastornos de personalidad diagnosticados con los criterios establecidos por el eje II del DSM-IV y la motivación para iniciar un tratamiento. En una muestra de 1083 pacientes admitidos en una variedad de programas psicoterapéuticos, encontraron que pacientes con trastornos de personalidad mostraron una motivación significativamente mayor al tratamiento que aquellos pacientes que no cumplían con los criterios para ser diagnosticados con dichos trastornos. Esta asociación aparentemente podría ser explicada por el elevado nivel de síntomas molestos que presentarían estos pacientes.

En un estudio cuyo objetivo fue determinar el impacto negativo de la presencia de trastorno de personalidad en los resultados de la terapia cognitiva conductual multimodal para pacientes con desorden obsesivo-compulsivo, se encontró que un porcentaje importante de los pacientes se benefició del tratamiento independiente de la presencia o no de un trastorno de personalidad. Sin embargo, algunos rasgos de personalidad específicos (esquizotípicos, pasivoagresivos) fueron determinantes de fracasos posteriores del tratamiento (Fricke et al., 2006).

En otro estudio se investigó la manera como las características de sujetos con trastornos de personalidad limítrofe predecían las variaciones en los resultados terapéuticos a dos años de seguimiento. Los resultados confirmaron hallazgos anteriores que señalan que mayores niveles de severidad del trastorno de personalidad limítrofe predicen resultados terapéuticos más pobres (Gunderson et al., 2006).

Newton-Howes, Tyrer y Johnson (2006), en un metaanálisis sobre los resultados psicoterapéuticos (recuperación/no recuperación) en pacientes depresivos con y sin trastorno de personalidad, encontraron que la comorbilidad de un trastorno de personalidad con trastorno depresivo se asocia con el doble de riesgo de magros resultados para el tratamiento de la depresión. Sin embargo, Hilsenroth, Defife, Blake y Cromer (2007), en un estudio sobre el efecto del trastorno de personalidad limítrofe sobre los resultados de la terapia psicodinámica breve para la depresión, encontraron que los pacientes con y sin trastorno de personalidad tuvieron mejoras sustanciales para la sintomatología depresiva, siendo las diferencias entre ambos grupos el momento de la terapia en el cual los pacientes mostraron dichas mejorías. Los pacientes sin trastorno de personalidad limítrofe mostraron mejorías antes de la novena sesión, mientras que los pacientes con trastorno de personalidad limítrofe mostraron mejorías después de la novena sesión.

Por su parte, Weertman, Arntz, Schouten \& Dreessen (2005) investigaron los efectos de los trastornos de personalidad, y de algunas creencias específicas relacionadas con éstos, sobre los resultados de la terapia cognitivo-conductual para los trastornos de ansiedad en una muestra de 398 pacientes. Los resultados mostraron que los pacientes con trastornos de personalidad reportaron mayores niveles de síntomas una vez concluido el tratamiento. Sin embargo, este efecto no fue tan potente como podría haberse esperado sobre las bases de estudios anteriores.

Otro rasgo de personalidad que ha sido estudiado por su influencia en el proceso y los resultados terapéuticos ha sido el de las expectativas del paciente. Frank (1979) consideraba que la confianza de los pacientes en sus terapeutas 
y en el tratamiento era un determinante crítico de los resultados de la psicoterapia. También se ha planteado que las expectativas del paciente se relacionan con la adhesión y duración de la terapia (Clarkin \& Levi, 2004).

En un estudio cuyo objetivo era evaluar el grado en que las expectativas del paciente respecto del tratamiento predecían los resultados a corto y largo plazo de una terapia cognitivo-conductual para el dolor crónico, se encontró que las expectativas antes del tratamiento predecían significativamente los resultados medidos inmediatamente después de terminado el tratamiento y en el seguimiento a los doce meses (Goossens et al., 2005). Sin embargo, en otra investigación realizada en adultos tratados por trastorno obsesivo-compulsivo, se encontró que las expectativas sobre el tratamiento no se relacionaron significativamente con los resultados del mismo, a diferencia de lo que ocurría con la alianza terapéutica (Vogel, Hansen, Stiles \& Götestam, 2007). Al respecto, en otra investigación se trabajó con la hipótesis de que la alianza terapéutica podía ser una variable mediadora entre las expectativas del paciente y los resultados de la terapia. Se estudiaron dos grupos psicodinámicos de tiempo limitado y se encontró que la asociación entre expectativa y resultado se expresaba a través del desarrollo de una relación de colaboración entre los miembros del grupo y con el terapeuta (Abouguendia, Joyce, Piper \& Ogrodniczuk, 2004).

En una revisión sobre las expectativas de los pacientes como un factor común de la psicoterapia, Dew y Bickman (2005) concluyeron que las expectativas de los pacientes se relacionan con los resultados del tratamiento y con la alianza terapéutica, pero que no se cuenta con evidencia suficiente para apoyar la relación entre las expectativas de los pacientes y los conflictos con el tratamiento.

La fuerza del ego es otra variable de personalidad que ha sido estudiada en relación con su efecto en el proceso y los resultados psicoterapéuticos (Garfield, 1994). La fuerza del ego es definida como la presencia de activos positivos en la personalidad que le permiten al individuo tolerar y sobrellevar sus ansiedades y adquirir mecanismos de defensas más adecuados (Clarkin \& Levin, 2004).

En un meta-análisis, Meyer y Handler (1997) concluyeron que la fuerza del ego medida por el RPRS (Rorschach Prognostic Rating Scale) es un buen predictor de los resultados de la psicoterapia. Por su parte, Clemence (2004) investigó la efectividad del RPRS para predecir los resultados de la psicoterapia empleando una base de datos de 90 pacientes seriamente perturbados tratados con psicoterapias intensivas. Los resultados de este estudio aportaron evidencia a la validez predictiva del constructo fortaleza del ego respecto de los resultados de la psicoterapia.

Finalmente, la capacidad de mentalización ha sido señalada como otro aspecto de la personalidad relacionado con los resultados psicoterapéuticos. McCallum y Piper
(1996) la han definido como la habilidad de comprender a las personas y sus problemas en términos psicológicos.

En un estudio se exploró la fortaleza relativa de dos características del paciente, la capacidad de mentalización y la alexitimia, como predictoras de los resultados psicoterapéuticos. Se encontró una relación directa y significativa entre el nivel de mentalización y los resultados favorables de las terapias (McCallum, Piper, Ogrodniczuk \& Joyce, 2003).

Clarkin \& Levy (2004) han hipotetizado que los pacientes con altos niveles de mentalización se beneficiarían más de terapias interpretativas en las cuales los conflictos internos suelen ser explorados, mientras que los pacientes con bajos niveles de mentalización serían mejores trabajando y beneficiándose de las terapias de apoyo en las cuales los conflictos internos no son explorados.

En un estudio que evaluó el nivel de mentalización de los pacientes en tres intervenciones para la depresión adolescente (terapia cognitivo-conductual, terapia familiar sistémica y terapia de apoyo no directivo) se encontró que puntajes altos de mentalización no fueron predictores de mejorías en la sintomatología depresiva. Sin embargo, los resultados mostraron una interacción entre el nivel de mentalización y la respuesta a los distintos tratamientos a lo largo del tiempo. Los pacientes en terapia cognitivoconductual con mayores niveles de mentalización mostraron una mejoría más rápida comparados con los pacientes de terapia familiar sistémica (Boylan, 2006). Piper et al. (2001), en una investigación similar, estudiaron la interacción de dos características de personalidad de los pacientes (calidad de las relaciones objetales y mentalización) con dos formas de terapias grupales de tiempo limitado (interpretativa y de apoyo). Para el nivel de mentalización sólo encontró un efecto principal: los pacientes con altos niveles de mentalización mostraron más mejorías en ambos tipos de terapia.

Esta revisión, sin pretender agotar todas las variables de personalidad de los pacientes investigadas por sus efectos en el proceso y los resultados terapéuticos, permite ilustrar dos aspectos del estudio de la personalidad como una variable independiente del proceso psicoterapéutico.

Un primer aspecto a destacar es que esta aproximación parece concitar mayor interés entre los investigadores que el estudio del cambio de la personalidad como efecto de la psicoterapia. Como se puede apreciar, es posible referir un mayor número de investigaciones y más actualizadas respecto de la relación entre las variables de personalidad de los pacientes y sus efectos en el proceso y los resultados terapéuticos.

Un segundo aspecto, esta vez relacionado con las concepciones sobre la personalidad a la base de estas investigaciones, es que se asume que las variables de personalidad que afectan el proceso y los resultados terapéuticos se caracterizan por cierto grado de estabilidad. Así, 
por ejemplo, la presencia de un trastorno de personalidad, las expectativas del paciente respecto del tratamiento, el grado de fortaleza yoica o la capacidad de mentalización, suelen ser conceptualizadas como características inmutables de los pacientes y se las suele medir antes de iniciada la terapia para relacionarlas con los resultados de la misma. De esta forma, parece no considerarse que estas variables de la personalidad puedan verse alteradas a propósito de la psicoterapia y, en una relación de mutua influencia, impactar los resultados de la misma.

\section{Conclusiones y Discusión}

La investigación en psicoterapia ha cambiado desde sus orígenes y estos cambios han implicado una pérdida de centralidad de las teorías integradas de la personalidad como guía y criterio de evaluación de los resultados terapéuticos. Junto con lo anterior, las diferentes respuestas a la pregunta respecto de si la personalidad cambia o no, permiten la distinción entre diferentes modelos de la personalidad, con diferentes implicancias teóricas, metodológicas y prácticas de relevancia para su relación con el estudio de la efectividad de la psicoterapia.

Así, por ejemplo, si la respuesta es que la personalidad no cambia, entonces el valor del constructo para la investigación en psicoterapia no es el de una medida de resultado (e.g. variable dependiente), sino el de una variable que afecta el proceso y determina sus resultados (e.g. variable independiente). En términos metodológicos, los estudios se orientan al establecimiento de la relación entre las variables de personalidad de los pacientes (e.g.. expectativas sobre el tratamiento), algunas características relevantes del proceso terapéutico (p.e. alianza terapéutica) y los resultados terapéuticos (e.g. disminución de la sintomatología). De manera opuesta, si la respuesta es que la personalidad cambia, entonces el valor del constructo para la investigación en psicoterapia es el de una medida de resultado (e.g. variable dependiente). Los estudios se orientan, entonces, al establecimiento de indicadores confiables y clínicamente significativos de cambio de la personalidad (e.g. criterios DSM-IV eje II) y al establecimiento de los tiempos necesarios de tratamiento para que dichos cambios ocurran (e.g. Modelo dosis-efecto).

Sin embargo, las ideas sobre si la personalidad cambia o no cambia, no son necesariamente excluyentes. Por el contrario, es posible plantear que la personalidad está constituida por aspectos estables y aspectos que son susceptibles de ser modificados. Así, en relación con la investigación de la efectividad de la psicoterapia cabría considerar que algunas variables de personalidad de los pacientes podrían cambiar como efecto de la psicoterapia $\mathrm{y}$, en una relación de influencia recíproca, otras variables o las mismas impactar el curso del proceso terapéutico y los resultados del mismo.
Los estudios de resultado que se focalizan en los efectos de la psicoterapia en la personalidad, han permitido establecer que algunas variables de la personalidad de los pacientes cambian en el tiempo y a lo largo del curso de la psicoterapia. No obstante, también han señalado otras variables de personalidad que muestran consistencia en el tiempo (e.g. rasgos de personalidad). En este contexto, los instrumentos e indicadores que permiten evaluar el cambio en la personalidad son variados y no todos ellos permiten dar cuenta de esta complejidad. Si bien un análisis crítico de los instrumentos de medición y caracterización de la personalidad escapa a los propósitos de este artículo, baste con subrayar los cuestionamientos que en este sentido se han hecho a la validez del Eje II del DSM-IV como sistema clasificatorio de los trastornos de personalidad.

Aún cuando se ha establecido la posibilidad de modificación de la personalidad como efecto de la psicoterapia, la investigación de esta relación, por un lado, y la generalización de estos efectos en la práctica, por otro lado, se encuentran con la dificultad de que la medición y logro de estos resultados depende directamente de la duración de la psicoterapia. En este sentido, el énfasis de la evaluación de la efectividad de la psicoterapia en términos de la disminución de la sintomatología (p. e. Eje I DSM-IV), con una mayor importancia asignada a los cambios observables y la emergencia y popularidad de las psicoterapias breves, parecen obstaculizar un mayor desarrollo de la investigación de la modificación de la personalidad como una aspecto de la efectividad psicoterapéutica.

Por último, debido a que son pocos los estudios en esta área, los que además han privilegiado una aproximación naturalista ( $\sin$ grupo control), no se puede sostener con certeza que los cambios de la personalidad ocurridos en el curso de la psicoterapia pueden explicarse de manera significativa por dicha intervención y, además, no se han validado empíricamente técnicas específicas que operen como mecanismos de dichos cambios.

Con todo, esta aproximación al estudio de la relación entre psicoterapia y personalidad, se nos presenta como un área en incipiente desarrollo y a la vez con un evidente potencial para la producción de modelos validados empíricamente que contribuyan a una mayor efectividad de la psicoterapia.

Por su parte, los estudios de proceso y proceso-resultado que se orientan a estudiar las variables de personalidad de los pacientes que afectan el proceso y los resultados terapéuticos, han mostrado un mayor desarrollo y han aportado con conclusiones relevantes para la práctica psicoterapéutica. $\mathrm{Al}$ respecto, podría plantearse que, si bien estas variables de personalidad predicen ciertas características del proceso y ciertos resultados de la terapia, carecen de relevancia toda vez que no son variables que podamos manejar o intervenir en la práctica (no es ético ni conducente la selección de pacientes para la realización de la psicoterapia). Sin embargo, 
desde otra perspectiva, el conocimiento respecto de los efectos específicos de ciertas variables de personalidad sobre el proceso y los resultados terapéuticos puede ser relevante para el desarrollo de intervenciones particulares que contribuyan al cambio. Un ejemplo de lo anterior es el establecimiento del efecto diferencial de las terapias interpretativas y de apoyo según la capacidad de mentalización de los pacientes. Aún cuando no se cuenta con suficiente evidencia respecto de esta relación, los resultados producidos hasta el momento indican la importancia de la capacidad de mentalizar de los pacientes para beneficiarse de las terapias, lo cual, en la práctica, implicaría la relevancia de evaluar esta variable de personalidad en los pacientes y ajustar las intervenciones terapéuticas para potenciar o contribuir con dicha relación.

\section{Referencias}

Abouguendia, M., Joyce, A., Piper, W. \& Ogrodniczuk, J. (2004). Alliance as a Mediator of Expectancy Effects in Short-Term Group Psychotherapy. Group Dynamics: Theory, Research, and Practice, 8, 3-12.

Asay, T. \& Lambert, M. (1999). The empirical case for the common factors in therapy: quantitative findings. En: M. Hubble, B. Duncan \& S. Miller. The heart and soul of change. What works in therapy. Washington, D.C.: American Psychological Association.

Bateman, A. \& Fonagy, P. (2008). Comorbid antisocial and borderline personality disorders: Mentalization-based treatment. Journal of Clinical Psychology, 64(2), 181-194.

Bergin, A. \& Lambert, M. (1978). The evaluation of therapeutic outcomes. En: A. Bergin \& S. Garfield (Edits.), Handbook of psychotherapy and behavior change. New York: Wiley.

Boylan, M. (2006). Psychological mindedness as a predictor of treatment outcome with depressed adolescents. Dissertation Abstracts International: Section B: The Sciences and Engineering, 67, 3479.

Clarkin, , J. \& Levi, K. (2004). The influence of client variables on psychotherapy. En: A. Bergin \& S. Garfield (Edits.), Handbook of psychotherapy and behavior change. New York: Wiley.

Clemence, A. (2004). Use of Rorschach variables in the assessment of psychotherapy progress. Dissertation Abstracts International: Section B: The Sciences and Engineering, 64, 4608.

Day, A. \& Bryan, J. (2007). Personality change and personality disorder: some initial thoughts on the application of McAdam's triarchic model to the treatment of personality disorder. Psychology, Crime \& Law, 13, 19-26.

De Fruyt, F., Van Leeuwen, K., Bagby, R. M., Rolland, J. \& Rouillon, F. (2006). Assesing and interpreting personality change and continuity in patients trated for major depresión. Psychological Assessment, 18(1), 71-80.

Dew, S., \& Bickman, L.(2005). Client Expectancies About Therapy. Mental Health Services Research, 7, 21-33.

Fonagy, P., Roth, A., Higgits, A. (2005). Psychodynamic psychotherapies: evidence-based practice and clinical wisdom. Bulletin of the Menninger Clinic, 69(1), 1-57.

Fowler, C., Ackermann. S, Speanburg, S., Bailey, A., Blagys, M. \& Concklin, A. (2004). Personality and symptom change in treatmentrefractory inpatients: Evaluation of The Phases Model of Change using Rorschach, TAT and DSM-IV axes V. Journal of Personality Assesment, 83, 306-322.

Fowler, J., Ackerman, S., Speanburg, S. Bailey, A. \& Blagys, M. (2004). Personality and symptom change in treatment-refractory inpatients: evaluation of the phase model of change using Rorscharch, TAT, and DSM-IV Axis V. Journal of personality assessment, 83, 306-322.
Frank, J. (1979). The present status of outcome studies. Journal of Consulting and Clinical Psychology, 47, 310-316.

Fricke, S., Moritz, S., Andresen, B., Jacobsen, D., Kloss, M., Rufer, M. \& Hand, I. (2006). Do personality disorders predict negative treatment outcome in obsessive-compulsive disorders? A prospective 6-month follow-up study. European Psychiatry, 21, 319-324.

Garfield, S (1994). Research on client variables in psychotherapy. En: A. Bergin \& S. Garfield (Edits.) Handbook of psychotherapy and behavior change. New York: Wiley.

Goossens, M., Vlaeyen, J., Hidding, A., Kole-Snijders, A., \& Evers, S. (2005). Treatment Expectancy Affects the Outcome of CognitiveBehavioral Interventions in Chronic Pain. Clinical Journal of Pain, $21,18-26$

Gunderson, J., Daversa, M., Grilo, C., McGlashan, T., Zanarini, M., Shea, M., Skodol, A., Yen, S., Sanislow, C., Bender, D., Dyck, I., Morey, L. \& Stout, R. (2006). Predictors of 2-Year Outcome for Patients With Borderline Personality Disorder. American Journal of Psychiatry, 163, 822-826.

Hilsenroth, M., Defife, J., Blake, M., \& Cromer, T. (2007). The effects of borderline pathology on short-term psychodynamic psychotherapy for depression. Psychotherapy Research, 17, 175-188.

Hoglend, P., Oslo, N., Bogwald, K., Amlo, S., Marble, A., Ulberg, R., Sjaastad, M., Sorbye, O., Heyerdahl, O., \& Johansson, P. (2008). American Journal of Psychiatry, 165, 763-771.

Howard, K., Moras, K., Brill, P., Martinovich, Z. \& Lutz, W. (1996). Evaluation of psychotherapy. Efficacy, effectiveness, and patient progress. American Psychologist, 51, 1059-1064.

Krause, M. (2005). Psicoterapia y cambio. Una mirada desde la subjetividad. Santiago de Chile: Ediciones Universidad Católica.

Lambert, M. \& Barley, D. (2001). Research summary on the therapeutic relationship and psycotherapy outcome. Psychoterapy: Theory, Research, Practice, Training, 38, 357-361.

Lambert, M. \& Supplee, E. (1997). Trends and practice in psychotherapy outcome assessment and their implicatios for psychotherapy and applied personality. En: R. Hogan, J. Johnson, S. Briggs. (Editores). Handbook of personality psychology. New York: Academic Press.

Lingiardi, V., Shedler, J. \& Gazzilio, F. (2005). Assesing personality change in psychotherapy with the SWAP-200: A case study. Journal of Personality Assesment, 86, 23-32.

Lingiardi, V., Shelder, J., Gazzillo, F. (2006). Assessing Personality change in psychotherapy with the Swap-200: a case study. Journal of Personality Assessment, 86, 23-32.

Mayer, J. (2004). How does psychotherapy influence personality? A theoretical integration. Journal of Clinical Psychology, 60(2), 1921-1315.

McCallum, M. \& Piper, W. (1996). Psychological mindedness. Psychiatry, $59,361-364$

McCallum, M., Piper, W., Ogrodniczuk, J., \& Joyce, A. (2003). Relationships among psychological mindedness, alexithymia and outcome in four forms of short-term psychotherapy. Psychology and Psychotherapy: Theory, Research and Practice, 76, 133-144.

Messer, S. \& Wampold, B. (2002). Lets's face the facts: common factors are more potent than specific therapy ingredients. Clinical Psychology, Science and Practice, 9, 21-25.

Meyer, G. J. \& Handler, L. (1997). The ability of the Rorscharch to predict subsequent outcome: A meta-analysis of the Rorschach Prognostic Rating Scale. Journal of Personality Assessment, 69, 1-38.

Millon, T., Meagher, S., Grossman, S. (2001). Theoretical perspectives. En: W. J. Livesley (Edit.) Handbook of personality disorders. New York: Guilford Press.

Newton-Howes, J., Tyrer, P., \& Johnson, T. (2006). Personality disorder and the outcome of depression: Meta-analysis of published studies. British Journal of Psychiatry, 188, 13-20.

Ogles, B., Lambert, M., Weight, D. \& Payne, I. (1990). Agoraphobia outcome measurement: A review and meta-analysis. Psychological Assessment, 2, 317-325.

Paris, J.(2005). Outcome and epidemiological research on personality disorders: implication for classification. Journal of Personality Disorders, 19, 557-562. 
Perry, C., Banon, E., \& Ianni, F. (1999). Effectiveness of psychotherapy for personality disorders. American Journal of Psychiatry, 156(9), 1312-1321.

Piper, W., McCAllum, M., Joyce, A., Rosie, J., \& Ogrodniczuk, J. (2001). Patient personality and time-limited group psychotherapy for complicated grief. International Journal of Group Psychotherapy, 51, 525-552.

Rogers, C. (1957). The necessary and sufficient conditions of therapeutic personality change. Journal of Consulting Psychology, 21, 95-103.

Roth, A., \& Fonagy, P. (2006). What works for whom? A critical review of psychotherapy research. (2nd ed.). New York: Guilford.

Skodol, A., Shea, M., Monrey, L., Bender, D., Zanarini, M. \& Pagano, M. (2005). The collaborative longitudinal personality disorders study (CLPS): Overview and implications. Journal of Personality Disorders, 19(5), 487-504.

Sprenkle, D., \& Blow A. (2004). Common factors and our sacred models. Journal of Marital and Family Therapy, 30, 113-129.

Tallman, K. \& Bohart, A. (1999). The client as a common factor: clients as self-healers. En: M. Hubble, B. Duncan \& S. Miller, The heart and soul of change. What works in therapy. Washington, D.C.: American Psychological Association.

Tickle, J., Heatherton, T., Wittenberg, L. (2001). Can personality change?. En W. J. Livesley (Edit.) Handbook of personality disorders. New York: Guilford Press. van Beek, N. \& Verheul, R. (2008). Motivation for treatment in patients with personality disorders. Journal of Personality Disorders, 22, 89100 .

Vogel, P., Hansen, B., Stiles, T., Götestam, K. (2007). Treatment motivation, treatment expectancy, and helping alliance as predictors of outcome in cognitive behavioral treatment of OCD. Journal of Behavior Therapy and Experimental Psychiatry, 37, 247-255.

Wampold, B. (2005). Establishing specificity in psychotherapy scientifically: desing and evidence issues. Clinical Psychology, Science and Practice, 12, 194-197.

Wampold, B., Ahn, H., Coleman, H. (2001). Medical model as metaphor: old habits die hard Journal of Counseling Psychology, 48, 268-273.

Weertman, A., Arntz, A., Schouten, E. \& Dreessen, L. (2005). Influences of beliefs and personality disorders on treatment outcome in anxiety patients. Journal of Consulting and Clinical Psychology, 73, 936-944.

Zanarini, M., Frankenburg, F., Hennen, J., Reich, D. \& Silk, K. (2005). The McLean Study of Adult Development (MASD): Overview and implications of the first six years of prospective follow-up. Journal of Personality Disorders, 19(5), 505-523. 